\title{
Uneventful Spinal Anesthesia in a Patient with Precipitous Drop of Platelet Secondary to HELLP Syndrome: A Case Report and Review of Literatures
}

\author{
Joe Z. Liu, Chunhua Li, Hong Wang
}

Department of Anesthesiology, Detroit Medical Center, Detroit, USA.

Email: liuz50@hotmail.com

Received July $5^{\text {th }}, 2012$; revised August $6^{\text {th }}, 2012$; accepted August $22^{\text {nd }}, 2012$

\begin{abstract}
Thrombocytopenia caused by Hemolysis, Elevated Liver enzymes and Low Platelet count (HELLP) syndrome in preeclampsia parturients can be associated with substantial maternal and neonatal morbidity. Data on the issue of the safety of neuraxial anesthesia with thrombocytopenia in HELLP syndrome is limited. A lower limit of 100,000 per microliter for platelet count was suggested as "safe" for performing neuraxial anesthesia, however there is no supporting data. This lower limit is challenged lately. We present a case of uneventful spinal anesthesia for urgent Cesarean section in a patient with severe pre-eclamsia, HELLP syndrome and precipitous platelet drop from 230,000 to 42,000 per microliter.
\end{abstract}

Keywords: Thrombocytopenia; Pre-Eclampsia; HELLP Syndrome; Parturient; Cesarean Section; Platelet; Neuraxial Anesthesia; Neuraxial Hematoma

\section{Introduction}

Pregnant women are usually young and healthy, and the expectation is that modern childbirth is relatively safe for mother and baby. Any intervention in childbirth, for example, to provide good analgesia, should carry minimal risk. Rare but serious adverse events are important in this circumstance.

Neuraxial hematoma is a devastating complication. It can lead to permanent neurological damage and paralysis. The negative impact on quality of life, together with the economic costs of a serious adverse event, is especially important in this patient group. The frequency of neuraxial hematoma in the obstetric population is unknown and difficult to determine. In larger post-1990 studies, the incidence of epidural hematoma was estimated to be 1 in 168,000 women, or 6 per million [1]. Among the published case reports of parturients who have experienced neuraxial hematoma after neuraxial blockade, a significant proportion of patients had altered coagulation at the time of block placement [2].

Thrombocytopenia caused by Hemolysis, Elevated Liver enzymes and Low Platelet count (HELLP) syndrome in pre-eclampsia parturients can be associated with substantial maternal and neonatal morbidity. Data on the issue of the safety of neuraxial anesthesia with thrombocytopenia is limited. A lower limit of 100,000 per microliter for platelet count was suggested as "safe" for per- forming neuraxial anesthesia, however there is no supporting data $[3,4]$. This lower limit is challenged lately.

Guidelines for the use of platelet transfusions [5] recommends that platelet count should be raised to at least 50,000 per microliter for lumbar puncture, and epidural anesthesia. However, this recommendation was given on the basis of expert opinion and a study of over 5,000 lumbar punctures in children with acute leukemia [6]. It is likely that lower platelet counts may also be safe but there is insufficient published evidence to make recommendations for lower levels at this stage.

For patients with platelet counts of 50,000 to 80,000 per microliter requiring epidural or spinal anesthesia and patients with a platelet count 20,000 to 40,000 per microliter requiring a lumbar puncture, an individual decision based on assessment of risks and benefits should be made [7].

Here we present a case of uneventful spinal anesthesia for urgent cesarean section in a patient with severe preeclamsia, HELLP syndrome and precipitous drop of platelet to 42,000 from 230,000 per microliter.

\section{Case Report}

A 21-year-old P1G0, body mass index of 40 was admitted at 29-5/7 weeks gestation for mild preeclampsia.

On day 1 of admission, her blood pressures were mildly elevated. Aspartate aminotransferase (AST) was 
$18 \mathrm{U} / \mathrm{L}$, creatinine $0.8(\mathrm{mg} / \mathrm{dL})$, platelet $230,000 / \mu \mathrm{L}$ and $1+$ protein on urine analysis. She showed no neurological signs or symptoms of preeclampsia.

On day 2, fetal status was reassuring. Fetal non-stress test was done daily. She received 2 doses of betamethasone. Her 24-hour urine showed total volume of $1225 \mathrm{ml}$, total protein of $502 \mathrm{mg}$. She was diagnosed with mild preeclampsia. At that time no anti-hypertensive medications were used.

On day 3 , her blood pressure started to rise. She gained $6 \mathrm{~kg}$ in 2 days. She was transferred to maternal special care unit with a diagnosis of severe preeclampsia. Labor was induced. Blood pressure ranged from $150 \mathrm{~s}-160 \mathrm{~s} /$ $100 \mathrm{~s}-110 \mathrm{~s} \mathrm{mmHg}$. She started to complain of severe right upper quadrant pain, blurred vision and photophobia. She also complained of nausea and vomiting. There were 3 emetic episodes. Her AST increased from 18 to $602 \mathrm{U} / \mathrm{L}$ (see Table 1).

Magnesium sulfate was started for seizure prophylaxis. AST continued to rise from 602 to $1969 \mathrm{U} / \mathrm{L}$; alanine transaminase (ALT) increased from 13 to 348 to $819 \mathrm{U} / \mathrm{L}$. Lactate dehydrogenase increased from 162 to 968 to $2123 \mathrm{U} / \mathrm{L}$. Creatinine remained stable. Platelets declined from 230,000 to 158,000 to $42,000 / \mu \mathrm{L}$, which was verified manually by slides. PT, PTT, INR and fibrinogen were in normal limit. She was diagnosed with severe preeclampsia with HELLP syndrome. Decision was made to proceed with delivery via cesarean section.

Before going to OR, the benefits and risks of general and regional anesthesia were discussed with patient and her family. Since she is obese and pregnant, regional anesthesia, particularly spinal anesthesia was still preferred to general anesthesia with endotracheal intubation. She was informed the risk of spinal hematoma. However, since there was no evidence of thrombocytopathy and other coagulation disorders, compared to general anesthesia, it was still a preferred. She agreed with the plan.

Preoperative airway exam showed intact teeth, good mouth opening, Mallampatti 3, decent neck range of motion.

After positioning her on the OR bed in a sitting position, a satisfactory subarachnoid block with bupivacaine $12 \mathrm{mg}$ and preservative free morphine $0.2 \mathrm{mg}$ was achieved at first attempt by using a $25 \mathrm{G}$ Whitacre needle.

Table 1. Platelet count, AST and ALT changes during progression of preeclampsia.

\begin{tabular}{llll}
\hline & Day 1 & Day 3 & Day 4 \\
\hline Platelet count $(/ \mu \mathrm{L})$ & 230,000 & 158,000 & 42,000 \\
AST $(\mathrm{U} / \mathrm{L})$ & 18 & 602 & 1969 \\
ALT $(\mathrm{U} / \mathrm{L})$ & 13 & 348 & 819 \\
\hline
\end{tabular}

CSF was clear without bleeding.

Blood pressure (BP) was stable during surgery. Estimated blood loss at $1200 \mathrm{~mL} .2000 \mathrm{~mL}$ crystalloid was given. No blood products were used.

A 799 gm healthy male baby was delivered by cesarean section. Apgar scores were 5 and 8 at one and five minutes respectively.

Upon arrival at PACU, she was able to move both lower extremities.

Her liver enzymes came down soon after cesarean section, and her platelets rose above $100,000 / \mu \mathrm{L} 3$ days later.

She was discharged home on day 10. Careful neurological surveillance did not find any complications from spinal anesthesia.

\section{Discussion}

The risk factors of spinal hematoma associated with neuraxial anesthesia include anticoagulant and antiplatelet use, bleeding disorders, traumatic/difficult puncture, old age and abnormal anatomy of spine [2]. Very few cases were associated with thrombocytopenia alone [8-10]. Different causes of thrombocytopenia may have different risks of bleeding.

The lowest safe limit of platelet count has not yet been determined. One retrospective study of 2929 consecutive parturients found 24 women with thrombocytopenia $(15,000-99,000 /$ microliter). Fourteen of the 24 thrombocytopenic patients received regional anesthesia (12 epidurals, 2 spinals), and none had permanent sequelae [11].

One case report of a full term parturient who had severe unrecognized profound thrombocytopenia of platelet count of 2,000 per microliter did not have any neurological sequelae or excessive bleeding after continuous epidural placement [8].

Beilin, et al. in their retrospective review reported safe epidural analgesia in thirty parturients with platelet counts between 69,000 and 98,000 per microliter [9].

Several recent studies suggest that platelet counts above $40,000-80,000$ per microliter are acceptable to administer regional anesthesia provided normal platelet function and absence of coagulopathy [5-7,11,12]. The bleeding risk for spinal is likely to be much less than the bleeding risk of an indwelling epidural catheter [2].

Thrombocytopenia occurs in $50 \%$ of pre-eclamptic patients and occasionally precedes other manifestations of the disease. The thrombocytopenia is usually moderate and clinical hemorrhage is uncommon unless the patient develops disseminated intravascular coagulopathy. A decreasing maternal platelet count is considered as an early sign of worsening of preeclampsia and may occur even before other clinical manifestations of the disease are 
apparent. The pathogenesis of thrombocytopenia in women with severe preeclampsia is unknown, although vascular endothelial damage, impaired prostacyclin production and increased deposition of fibrin within the vascular bed have been suggested $[13,14]$.

The management of pre-eclampsia and HELLP syndrome is focused on stabilizing the mother until fetal maturity is more favorable. Fetal monitoring is mandatory, and will help to decide whether early delivery of the fetus is warranted. Early delivery of the fetus is warranted if there are threats to fetal or maternal life or if the syndrome develops beyond 34 weeks of gestation. Caesarean section is required in $60 \%$ of cases [15].

A retrospective study demonstrated that combined spinal-epidural anesthesia can be used in cases of HELLP syndrome [16]. Two patients received a combined spinal epidural although their immediate preoperative thrombocyte count was $<50,000$ per microliter.

The platelet count in patient with HELLP syndrome may fall precipitately and coagulopathy may develop quickly. Patients with HELLP syndrome in whom an intrapartum platelet count above 40,000 per microliter are unlikely to have clinically significant postpartum bleeding [17].

A decreasing platelet count is considered a contraindication to neuraxial blockade, especially in dynamic conditions such as pre-eclampsia and ITP. Pseudo-thrombocytopenia must be excluded. A manual platelet count is more accurate in patients with recent thrombocytopenia because automated counters are not reliable at low platelet counts [9].

To reduce the risk of hemorrhage and neurological complications the British Committee for Standards in Hematology recommends the use of prophylactic platelet transfusions if the platelet count is less than 40,000 per microliter in children [18] and if less than 50,000 per microliter in adults [5]. Spinal hemorrhage is rare and in both guidelines it is acknowledged that there is a lack of evidence to support the recommendations. The evidence for prophylactic transfusions is based on case reports of spinal hemorrhage following lumbar puncture.

Platelet transfusions have not been shown to reduce the risk of complications and there is evidence that lumbar punctures can be performed safely even in children with severe thrombocytopenia [6].

Assessment of the incidence of a rare event is difficult in clinical practice. Therefore, in the absence of large, unfeasible studies, it is prudent to evaluate the risk-benefit ratio on a case-by-case basis before administering regional anesthesia to parturients [19].

The unique aspect of our patient is the dynamic sudden drop of platelet. It is believed to be more dangerous than chronic stable thrombocytopenia. However, there is no report of spinal hematoma in this situation.

It was a challenging decision with drastic falling platelet count from 230,000 to 42,000 per microliter and possible difficult airway in this morbidly obese patient. The absent clinical bleeding and normal laboratory tests helped us to conclude that the benefit of avoiding risks associated with difficult airway in general anesthesia outweighs the risks of hemorrhagic complications from spinal anesthesia.

A physical examination of the patient should include looking for evidence of bruising and bleeding at venopuncture sites or petechiae at the blood pressure cuff site. Consumptive coagulopathy associated with placental abruption and other conditions must be ruled out. When considering regional anesthesia in patients with thrombocytopenia, spinal anesthesia may be safer. Careful monitoring of the patient in the postpartum period to detect early signs and symptoms of an epidural hematoma should be undertaken. General anesthesia for urgent cesarean section becomes necessary if coagulation is abnormal or there is bleeding [14].

\section{REFERENCES}

[1] W. Ruppen, S. Derry, H. McQuay and R. A. Moore, "Incidence of Epidural Hematoma, Infection, and Neurologic Injury in Obstetric Patients with Epidural Analgesia/Anesthesia," Anesthesiology, Vol. 105, No. 2, 2006, pp. 394399. doi:10.1097/00000542-200608000-00023

[2] T. Horlocker, D. J. Wedel, J. C. Rowlingson, et al., "Regional Anesthesia in the Patient Receiving Antithrombotic or Thrombolytic Therapy: American Society of Regional Anesthesia and Pain Medicine Evidence-Based Guidelines," Regional Anesthesia and Pain Medicine, Vol. 35, No. 1, 2010, pp. 64-101. doi:10.1097/AAP.0b013e3181c15c70

[3] Bromage, "Neurologic Complications of Regional Anaesthesia for Obstetrics," 3rd Edition, Williams \& Wilkins, 1993.

[4] S. H. Rolbin, E. Musclow, et al., "Epidural Anesthesia in Pregnant Patients with Low Platelet Counts," Obstetrics \& Gynecology, Vol. 71, No. 6, 1988, pp. 918-920.

[5] British Committee for Standards in Haematology, "Guidelines for the Use of Platelet Transfusions," British Journal of Haematology, Vol. 122, No. 1, 2003, pp. 10-23. doi:10.1046/j.1365-2141.2003.04468.x

[6] S. Howard, A. Gajjar, R. C. Ribeiro, et al., "Safety of Lumbar Puncture for Children with Acute Lymphoblasticleukemia and Thrombocytopenia," Journal of the American Medical Association, Vol. 284, No. 17, 2000, pp. 22222224. doi:10.1001/jama.284.17.2222

[7] J. van Veen, T. J. Nokes and M. Makris, "The Risk of Spinal Haematoma Following Neuraxial Anaes-Thesia or Lumbar Puncture in Thrombocytopenic Individuals," British Journal of Haematology, Vol. 148, No. 1, 2010, pp. 15-25. 


\section{doi:10.1111/j.1365-2141.2009.07899.x}

[8] P. Hew-Wing, S. H. Rolbin, E. Hew, et al., "Epidural Anaesthesia and Thrombocytopenia," Anaesthesia, Vol. 44, No. 9, 1989, pp. 775-777. doi:10.1111/j.1365-2044.1989.tb09270.x

[9] Y. Beilin, J. Zahn and M. Comerford, "Safe Epidural Analgesia in Thirty Parturients with Platelet Counts between 69,000 and 98,000 $\mathrm{mm}^{-3}$," Anesthesia and Analgesia, Vol. 85, No. 2, 1997, pp. 385-390.

[10] S. Choi and R. Brull, "Neuraxial Techniques in Obstetric and Non-Obstetric Patients with Common Bleeding Diatheses," Anesthesia and Analgesia, Vol. 109, No. 2, 2009, pp. 648-660. doi:10.1213/ane.0b013e3181ac13d1

[11] K. T. Rasmus, R. L. Kotelko, et al., "Unrecognized Thrombocytopenia and Regional Anesthesia in Parturients: A Retrospective Review," Obstetrics \& Gynecology, Vol. 73, No. 6, 1989, pp. 943-946.

[12] C. A. Schiffer, K. C. Anderson, C. L. Bennett, et al., "Platelet Transfusion for Patients with Cancer: Clinical Practice Guidelines of the American Society of Clinical Oncology," Journal of Clinical Oncology, Vol. 19, No. 5, 2001, pp. 1519-1538.

[13] K. McCrae, P. Samuels and A. D. Schreiber, "Pregnancy Associated Thrombocytopenia: Patho-Genesis and Management," Blood, Vol. 80, No. 11, 1992, pp. 2697-2714.

[14] P. Kam, S. A. Thompson and A. C. S. Liew, "Thrombo- cytopenia in the Parturient," Anaesthesia, Vol. 59, No. 3, 2004, pp. 255-264. doi:10.1111/j.1365-2044.2004.03576.x

[15] B. M. Sibai, "The HELLP Syndrome (Hemolysis, Elevated Liver Enzymes and Low Platelets). Much Ado about Nothing?" American Journal of Obstetrics and Gynecology, Vol. 162, No. 2, 1990, pp. 311-316.

[16] S. Palit, G. Palit, M. Vercauteren and Y. Jacquemyn, "Regional Anaesthesia for Primary Caesarean Section in Patients with Preterm HELLP Syndrome: A Review of 102 Cases," Clinical \& Experimental Obstetrics \& Gynecology, Vol. 36, No. 4, 2009, pp. 230-234.

[17] W. Roberts, K. G. Perry Jr., J. B. Woods, et al., "The Intrapartum Platelet Count in Patients with HELLP (Hemolysis, Elevated Liver Enzymes, and Low Platelets) Syndrome: Is It Predictive of Later Hemorrhagic Complications?" American Journal of Obstetrics \& Gynecology, Vol. 171, No. 3, 1994, pp. 799-804.

[18] British Committee for Standards in Haematology, "Transfusion Guidelines for Neonates and Older Children," British Journal of Haematology, Vol. 124, No. 4, 2004, pp. 433-453. doi:10.1111/j.1365-2141.2004.04815.x

[19] V. Frenk, V. Camann and K. B. Shankar, "Regional Anesthesia in Parturients with Low Platelet Counts," Canadian Journal of Anesthesia, Vol. 52, No. 1, 2005, p. 114. doi:10.1007/BF03018594 